\title{
EFECTOS DE LA CRISIS ECONÓMICA EN LAS BIBLIOTECAS ESPAÑOLAS
}

\author{
Hilario Hernández-Sánchez y Natalia Arroyo-Vázquez
}

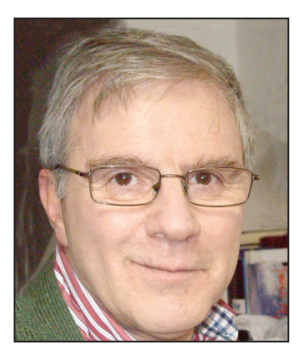

Hilario Hernández-Sánchez, director de Análisis y Estudios de la Fundación Germán Sánchez Ruipérez, es licenciado en geografía e historia y con estudios de biblioteconomía y documentación por la Universidad de Salamanca. Participa habitualmente en cursos de formación y foros profesionales y es autor de publicaciones sobre bibliotecas públicas, la lectura y la gestión de servicios culturales. Director de estudios sobre la situación y evolución de las bibliotecas públicas en España (2001, 2003, 2008 y 2009) promovidos por el Ministerio de Cultura y el Proyecto Tibidabo. Bibliotecas Públicas en Ciudades y Regiones de Europa. Desde la FGSR ha dirigido el proyecto Observatorio del Libro y la Lectura de Extremadura y varios estudios para Castilla y León, Castilla-La Mancha, Andalucía y Euskadi. Es presidente del Comité Científico del Observatorio de la Lectura de Andalucía. http://orcid.org/0000-0001-5510-5297

Análisis y estudios Fundación Germán Sánchez Ruipérez http://www.directorioexit.info/ficha2937

hilario.hernandez@fundaciongsr.es

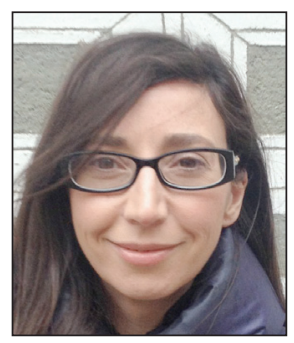

Natalia Arroyo-Vázquez es responsable de medios sociales en el del Área de Comunicación Digital de la Fundación Germán Sánchez Ruipérez desde 2012. Anteriormente ha estado vinculada a esta institución como documentalista en el Departamento de Análisis y Estudios. Autora del libro Información en el móvil y del Informe APEI sobre movilidad, participa en encuentros, congresos y otros foros profesionales y ha publicado varios artículos y comunicaciones. Tiene una amplia experiencia impartiendo formación especializada al personal de bibliotecas y centros de documentación en el uso de los medios sociales y de dispositivos móviles en la biblioteca. Es miembro del Grupo ThinkEPI y publica habitualmente en el blog Biblioblog. Es licenciada en documentación por la Universidad de Salamanca, donde actualmente completa sus estudios de doctorado.

http://orcid.org/0000-0002-4692-3420

Área de Comunicación Digital Fundación Germán Sánchez Ruipérez http://www.directorioexit.info/ficha820 narroyo@fundaciongsr.es

\section{Resumen}

Las bibliotecas en España, como otras instituciones, se están viendo afectadas por la crisis económica. Se analiza, a la luz de los datos estadísticos disponibles, en qué manera y cuáles son sus primeras consecuencias. Además de la importante disminución del gasto en adquisiciones y la consecuente desactualización de las colecciones, en 2012 se observa la desaparición de puntos de servicio y la reducción del personal, a la vez que se registra una creciente demanda de los servicios de biblioteca. También se pone de manifiesto el cambio de hábitos de consumo de contenidos y se presta una especial atención a lo digital, otro de los rasgos que más afectan a las bibliotecas en la actualidad. Datos de 2012: los gastos corrientes de las bibliotecas españolas ascendieron a 990,1 millones de euros (+0,54\% sobre 2010), aunque en realidad es $-5 \%$, si se tiene en cuenta el incremento del IPC. El gasto de personal representa un 66,9\%. Las bibliotecas públicas (BPs) registran 594 empleados menos que en 2010 y las universitarias 154 (-5,6\% y -2,6\% respectivamente). Las BPs incorporaron 3,5 millones de volúmenes (-31,2\% / 2010). Visitas: total 216,4 millones (BPs +20,5\% / 2010). 401 bibliotecas (20\%) no disponían de ordenadores para uso público y $46,5 \%$ bibliotecas estaban aún sin opac.

\section{Palabras clave}

Bibliotecas, Crisis económica, Bibliotecas públicas, Bibliotecas universitarias, Colecciones, Personal, Adquisiciones, Utilización, Visitas, Préstamos, Gastos, Estadísticas, España, Estadísticas bibliotecarias.

\section{Title: Effects of the economic crisis on Spanish libraries}

\begin{abstract}
Libraries in Spain, like other institutions, have been affected by the economic crisis. The effects and their consequences are studied in this paper, based on available library statistics. A significant decrease in expenditure for materials, the downgra-
\end{abstract}


ding of collections, library closures and a growing increase in usage were observed in 2012. Changes in content consumption habits are also highlighted, with special attention to adaptations to digital change. Facts 2012: the operating costs of Spanish libraries amounted to 990.1 million euros (+0.54\% compared to 2010); if the increase in the CPI is taken into account, the cost increase is actually 5\%. Personnel expenses represent 66.9\%. Public libraries (PLs) reported 594 fewer employees than in 2010 and there were 154 fewer in academic libraries (-5.6\% and $-2.6 \%$, respectively). The PLs incorporated $3.5 \mathrm{mi}-$ Ilion volumes (-31.2\% compared to 2010). Visits to libraries: Total 216.4 million (in PLs +20.5\% / 2010); 401 libraries (20\%) did not have computers for public use and $46.5 \%$ had no opac yet.

\section{Keywords}

Libraries, Public libraries, Academic libraries, Economic crisis, Librarians, Acquisitions, Expenditures, Usage, Visits, Loans, Statistics, Spain, Library statistics

Hernández-Sánchez, Hilario; Arroyo-Vázquez, Natalia (2014). "Efectos de la crisis económica en las bibliotecas españolas". El profesional de la información, marzo-abril, v. 23, n. 2, pp. 158-164.

http://dx.doi.org/10.3145/epi.2014.mar.08

\section{Introducción}

Es evidente que las bibliotecas en España, al igual que en otros países, se están viendo afectadas por la crisis económica global. La bibliografía refleja la preocupación por los recortes presupuestarios en estados de la cuestión más amplios sobre determinados tipos de bibliotecas (CarriónGútiez, 2013) y analizando aspectos concretos como la reacción de los profesionales (Gómez-Hernández, 2010), a través de experiencias de las propias bibliotecas (MerloVega, 2013), en estudios sobre el impacto en determinadas comunidades autónomas (Gutiérrez-Santana et al., 2010) o la necesidad de poner en valor sus servicios con estudios sobre el retorno de la inversión en bibliotecas como el recientemente publicado por Fesabid (Gómez-Yáñez, 2014).

La demanda de los servicios bibliotecarios no ha dejado de crecer en la última década, incluso se ha acentuado durante los años en que se produce la crisis económica

En otros países, las consecuencias de la crisis económica mundial son también visibles. En el Reino Unido se cerraron más de 200 bibliotecas públicas en el año fiscal 2011-2012 como consecuencia de los recortes; el personal se ve sustituido por voluntarios y se observa una tendencia descendente en el uso de las bibliotecas (Anstice, 2013). Los recortes también se han hecho notar en EUA desde 2009 tanto en el personal y los horarios de apertura como en las colecciones, servicios tecnológicos y salarios $(A L A, 2013)$.

El futuro de las bibliotecas se ha convertido en uno de los temas más recurrentes, aunque desde un punto de vista más tecnológico, y es que el cambio digital abre nuevos frentes en la configuración de los servicios bibliotecarios de los próximos años. Ambos aspectos, la economía y lo digital, definen a las bibliotecas en la actualidad.

La necesidad de contar con datos objetivos que pongan de manifiesto los efectos de los recortes presupuestarios y las consecuencias que están teniendo en las bibliotecas, así como la adaptación a la realidad digital, es la motivación de este trabajo. El tema, como señalaba Anglada (2012), es "cuánto bajarán y dónde se aplicarán los recortes", pero también qué consecuencias tendrán estos recortes sobre el sistema bibliotecario español. A partir de los últimos datos disponibles, correspondientes a 2012, ya se pueden avanzar algunas.

El precedente de este trabajo está en dos notas que se publicaron en el blog Biblioblog (Arroyo-Vázquez; HernándezSánchez; Villoldo; Villoldo, Hernández-Sánchez; ArroyoVázquez, 2012) y que ya avanzaban algunos de los puntos que se analizan aquí.

\section{El problema de las estadísticas de bibliotecas}

Los datos que publica el Instituto Nacional de Estadística $(I N E)^{1}$ con periodicidad bienal constituyen la fuente principal para el análisis de la situación de las bibliotecas en este trabajo. Estos datos se refieren a varios tipos de bibliotecas: Nacional, centrales de comunidades autónomas, públicas, para grupos especiales de usuarios, de instituciones de enseñanza superior y especializadas. Se ha tomado en esta ocasión la serie comprendida entre 2002 y 2012, ambos incluidos, siendo ese el último año del que se dispone de datos.

Como fuente complementaria se han estudiado los datos de bibliotecas públicas del Ministerio de Educación, Cultura y Deporte (MECD), que se publican cada año. En la web están disponibles los datos desde 2007 hasta 2011. Han sido completados con la serie de 2001 a 2005, obtenida del estudio Las bibliotecas públicas en España... (Hernández-Sánchez, 2008).

http://www.mcu.es/bibliotecas/MC/EBP/index.html

Si bien estas series estadísticas permiten estudiar la evolución de las bibliotecas a lo largo de toda una década, presentan varios problemas.

En primer lugar, son publicadas de forma parcial, lo que limita seriamente su análisis. En el caso del INE, no es posible consultar los datos por tipo de biblioteca y comunidad autónoma simultáneamente, excepto en los apartados de unidades administrativas y puntos de servicio, sino que sólo se muestran los datos totales para cada comunidad autónoma 


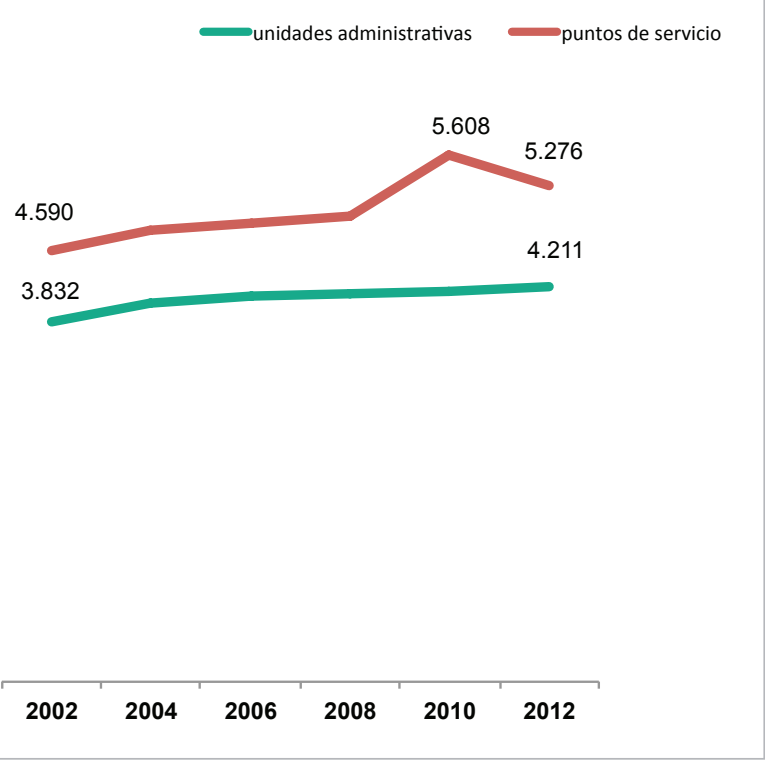

Gráfico 1. Evolución del número de puntos de servicio y unidades administrativas en bibliotecas públicas, 2002-2012.

o los datos totales por tipo de biblioteca. Estos son los niveles de desagregación más detallados que se proporcionan, a pesar de que cada biblioteca responde a la encuesta de forma individualizada.

En los datos del INE se han detectado además bailes contables en la clasificación de un grupo de bibliotecas (y sus puntos de servicio), que a lo largo de los últimos diez años se incluyen alternativamente entre las centrales de comunidades autónomas o entre las públicas, sin duda por su doble función, pero que generan en estos tipos oscilaciones artificiosas de un año a otro.

Por otra parte, algunas de las series están incompletas. Es notable la ausencia de la mayor parte de las bibliotecas de Catalunya en la serie del MECD en 2011, en la que se registran tan sólo los datos correspondientes a las tres bibliotecas públicas del Estado catalanas. El INE advierte de que las cifras sobre gastos corrientes sólo representan entre un $77,7 \%$ y un $64,6 \%$ de los casos, dependiendo de sus capítulos.

Teniendo esto en cuenta, así como otros aspectos puntuales que se citarán a lo largo del texto, es difícil manejar y analizar los datos económicos, algo que en la actual situación de crisis resulta absolutamente imprescindible, dado que es necesario contar con una información rigurosa para evaluar el coste del servicio, su eficacia y argumentar su posible mantenimiento ante los poderes públicos y ante la sociedad. Son los profesionales de las bibliotecas los primeros interesados en que esa información sea lo más fidedigna posible y totalmente transparente.

A todo ello hay que añadir que los datos no abarcan todas las áreas de actividad de las bibliotecas, como puede ser el uso de otros servicios diferentes del préstamo (lectura o estudio en sala, uso de los servicios de información y referencia, uso de recursos informáticos o asistencia a actividades), y que el último año del que se dispone información es 2012. Los datos relacionados con los servicios en línea constituyen un apartado especialmente mejorable, puesto que algunos

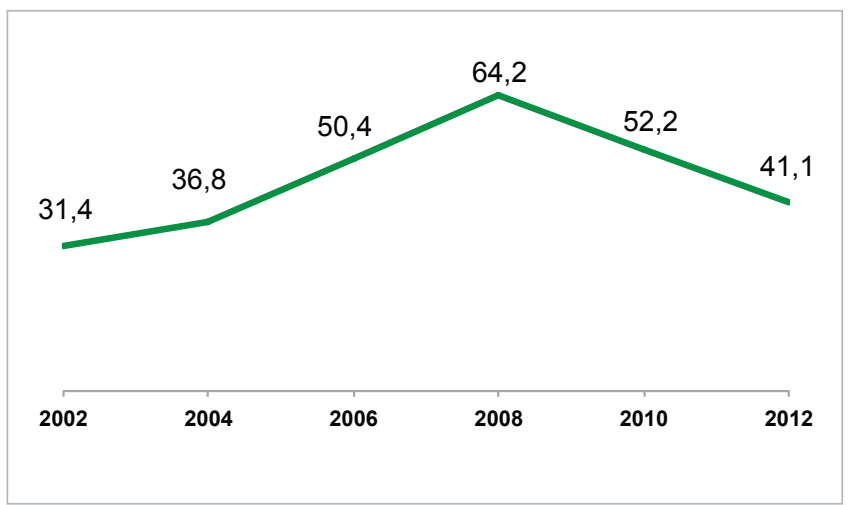

Gráfico 2. Gasto en adquisiciones en bibliotecas públicas, 2002-2012 (millones de euros).

aspectos no quedan reflejados, existen series incompletas y conceptos dudosos y sin embargo constituyen una parte cada vez más importante de la actividad de las bibliotecas. Todas estas limitaciones impiden obtener una visión completa y actual de la realidad de las bibliotecas de nuestro país, que debe ser dibujada con cautela tras un cuidadoso proceso de observación y tratamiento de los datos y, en ocasiones, con reservas a la hora de emitir conclusiones.

\section{La evolución de las bibliotecas en la última década}

\subsection{Acceso a los servicios bibliotecarios}

Las bibliotecas públicas constituyen el grupo más numeroso del sistema bibliotecario español. Sus 4.211 unidades administrativas en 2012 suponen el 61,9\% del total, lo que les concede un gran peso: los cambios que se producen en este grupo afectan notablemente al conjunto. Las bibliotecas especializadas son el siguiente grupo más numeroso, con 2.084 unidades administrativas. El 7,9\% restante lo componen bibliotecas universitarias (285), para grupos específicos de usuarios (240), centrales de comunidades autónomas (14) y la Biblioteca Nacional.

Las bibliotecas públicas constituyen el grupo más numeroso del sistema bibliotecario español: 4.211 unidades administrativas en 2012 que suponen el $61,9 \%$ del total

La crisis económica incide directamente en el número de puntos de servicio en 2012, año en que se registran 63 menos que en 2010. Esta reducción se debe fundamentalmente a la desaparición de 332 puntos de servicio de biblioteca pública, aunque una pequeña parte de la diferencia estaría provocada por ese baile contable explicado anteriormente. Sin embargo, los datos indican que en estos dos años el número de unidades administrativas se ha incrementado en 227 , lo cual se podría interpretar como una pérdida de puntos de servicio fundamentalmente en unidades administrativas con más de una biblioteca. 


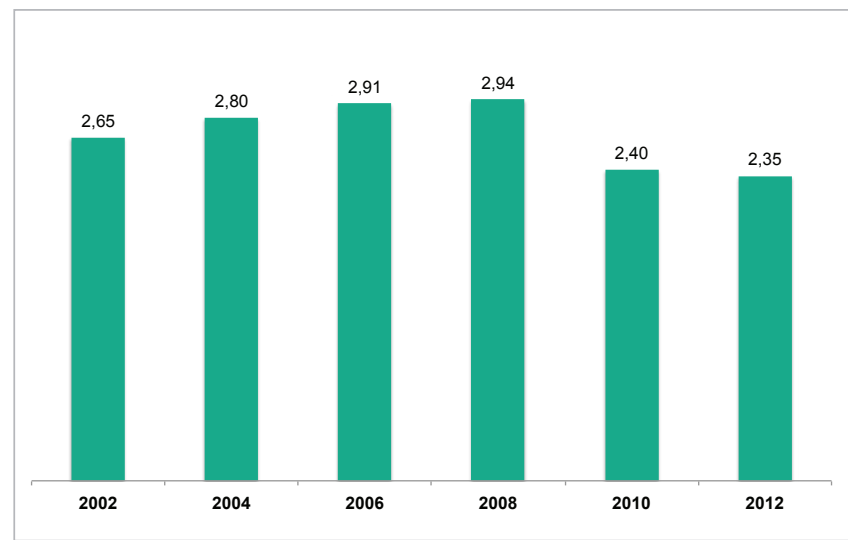

Gráfico 3. Empleados en equivalencia a tiempo completo por punto de servicio, 2002-2012.

La crisis también está incidiendo en la reducción de los horarios de las bibliotecas. Ha aumentado el número de bibliotecas con horarios de hasta 10 horas semanales (son en 2012 un 17,6\% más que en 2010) y se ha reducido ligeramente, en un $0,8 \%$, el número de bibliotecas con horarios superiores a las 40 horas semanales.

\subsection{El gasto en bibliotecas, lastrado por los recortes en adquisiciones}

En 2012 los gastos corrientes de las bibliotecas españolas ascendieron a 990,1 millones de euros, produciéndose un crecimiento del 0,54\% con respecto a los gastos de 2010 . Este mantenimiento ligeramente al alza de las asignaciones presupuestarias a las bibliotecas significa en realidad un decrecimiento en torno al $5 \%$, si se tiene en cuenta el incremento del IPC durante 2011 y 2012 (de 3,2 y 2,4 respectivamente, según recoge el INE).

Es en el capítulo de gastos de adquisiciones donde se observa un descenso generalizado desde 2010, y muy especialmente en las bibliotecas públicas, donde en 2012 se sitúa en niveles inferiores a los de 2006.

Los gastos de personal mantienen su crecimiento y llegan a representar un $66,9 \%$ del total en 2012. En bibliotecas públicas ese porcentaje se acentúa aún más, llegando al 74,0\% del gasto corriente, mientras que el gasto en adquisiciones se ve reducido al $8,7 \%$, lo que pone en evidencia cierto desequilibrio presupuestario. A modo de referencia, en 2008 el $15,6 \%$ de los gastos corrientes se destinaban a adquisiciones en las bibliotecas públicas y el $65,7 \%$ a personal.

Es llamativo el caso de la Biblioteca Nacional, que en 2012 consigna un gasto en adquisiciones del $5 \%$ de lo que se gastaba en 2002. El punto de inflexión se produce antes de la crisis, siendo 2008 el primer año que comienza a reflejar un descenso acusado.

\subsection{Personal bibliotecario}

El personal bibliotecario comienza a verse directamente afectado por la crisis económica en 2012, especialmente en las bibliotecas públicas y universitarias. Al descenso moderado en el número de personal se añade un aumento en el número de contratados a tiempo parcial en todo tipo de bibliotecas. Teniendo en cuenta que esta tendencia no es

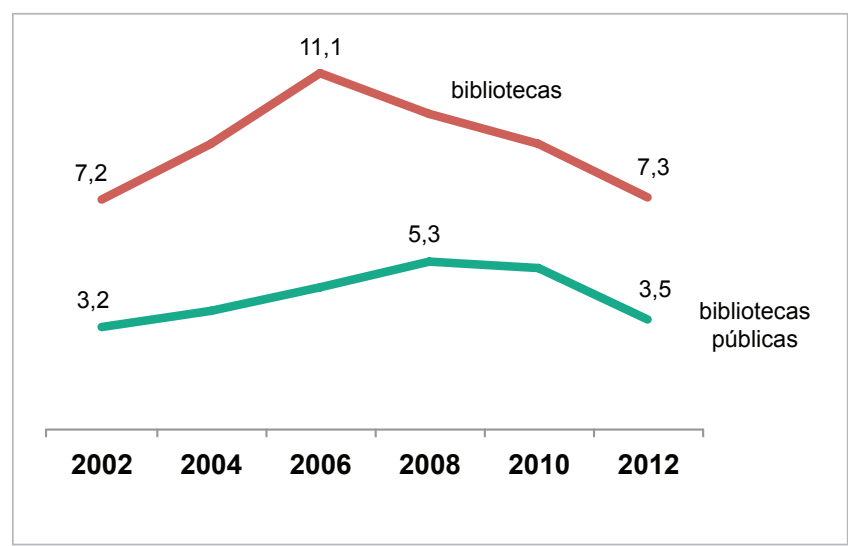

Gráfico 4. Evolución del número de adquisiciones de documentos en el conjunto de las bibliotecas y en bibliotecas públicas, 2002-2012 (millones).

paralela al gasto en personal, que por el contrario crece, se sospecha que se está incrementando la contratación a través de empresas, tal y como señalan Hernández-Sánchez, Gómez-Hernández y Merlo-Vega (2014).

En 2012 las bibliotecas públicas registran 594 empleados menos que en 2010 y las universitarias 154, lo que supone un descenso del 5,6\% y del 2,6\% respectivamente. En el caso de la Biblioteca Nacional fue 2010 el año en el que se rompió la tendencia al alza y se produce en 2012 una pérdida de 143 empleados en equivalencia a tiempo completo con respecto a 2008.

En 2012 se observa cómo la media de empleados por cada punto de servicio $(2,35)$ cae a su valor más bajo de los últimos diez años en el conjunto de las bibliotecas españolas. El aumento del número de contratos a tiempo parcial se deduce por la bajada en la tasa de equivalencia², que pasó de 0,86 en 2008 a 0,82 en 2012.

La disminución del número de adquisiciones es significativa en las bibliotecas públicas, que en 2012 registran incorporaciones por un total de 3,5 millones de volúmenes, un $31,2 \%$ menos que en 2010

\subsection{Las colecciones de las bibliotecas, desactualizadas}

La adquisición de documentos en las bibliotecas fue uno de los primeros capítulos en reflejar los signos de los recortes económicos, los datos de 2010 mostraban ya los primeros descensos. En 2012 la reducción en el número de adquisiciones continúa y se acentúa, de forma paralela a la importante reducción en el correspondiente capítulo del gasto, a pesar de lo que puede parecer a simple vista.

Los datos para el conjunto de las bibliotecas manifiestan un fuerte incremento en el número de adquisiciones en 2012, pero que es debido a la incorporación de más de seis millones de manuscritos en bibliotecas especializadas de archivos y museos en Andalucía y que representan el $44,5 \%$ 
de las altas a nivel nacional y en todas las bibliotecas. En 2010 ocurrió algo similar con el registro excepcional de 1,7 millones de libros electrónicos, la mayoría en bibliotecas especializadas en los centros de investigación madrileños. Si se obvian estos datos que producen efectos al alza que no se ajustan a la realidad general, se obtiene como resultado una reducción media anual del 7,9\% en el número de altas incorporadas a las bibliotecas entre 2008 y 2012.

El gran descenso en el número de adquisiciones lleva a pensar que las colecciones están envejeciendo

La disminución del número de adquisiciones es especialmente significativa en las bibliotecas públicas, que en 2012 registran incorporaciones por un total de 3,5 millones de volúmenes, casi la tercera parte menos (-31,2\%) que en 2010. Tras el descenso acumulado desde 2008, la incorporación de novedades en las bibliotecas públicas en 2012 alcanza valores similares a los de diez años antes, en 2002 (3,2 millones de volúmenes), y bastante lejos de los 4,5 millones registrados en 2006.

Las adquisiciones que más se han reducido son los documentos audiovisuales, sonoros y electrónicos, que acumulan un $35,4 \%$ de descenso entre 2008 y 2012 en el conjunto del sistema de bibliotecas. En el caso de las bibliotecas públicas el porcentaje es mayor y alcanza el $43,6 \%$. Pero también se observa una importante reducción en las altas de libros, que suponen una tercera parte menos de las registradas entre 2008 y 2012.

Las colecciones, por el contrario, no tendieron a la baja en 2012 , sino que muestran un ligero incremento. Aunque se podría interpretar de forma positiva que las bibliotecas públicas casi hayan alcanzado los 1,5 libros por persona que recomiendan las Directrices Ifla/Unesco para el desarrollo del servicio de bibliotecas públicas, el gran descenso en el número de adquisiciones lleva a pensar que las colecciones están envejeciendo.

\subsection{Crece la demanda de los servicios bibliotecarios}

La demanda de los servicios bibliotecarios no ha dejado de crecer en la última década, incluso se ha acentuado durante los años en que se produce la crisis económica, a partir de 2008, tal y como anticipó Gómez-Hernández (2010). El total de visitas recibidas en las bibliotecas en 2012 ascendió a 216,4 millones, cifra que aumenta ligeramente con respecto a 2010. Las bibliotecas públicas han registrado un importante incremento del número de visitas, el 20,5\% entre 2008 y 2012. El barómetro Hábitos... (2013) recoge además un incremento en la frecuencia de asistencia a bibliotecas entre 2010 y 2012. Sin embargo, el número de visitas en bibliotecas universitarias se redujo en un $5,4 \%$, siguiendo la tendencia a la baja iniciada ya en 2008.

El número de usuarios inscritos también va en aumento: en 2012 se suman 2,3 millones con respecto a 2010 (12,8\% más), de manera que el $43,2 \%$ de la población española estaría inscrita en alguna biblioteca. Este incremento es generalizado en prácticamente todo tipo de bibliotecas y en todas las comunidades autónomas.

Este dato no ha dejado de crecer a lo largo de la última década, con un incremento medio anual de $6,2 \%$ en los últimos diez años, si bien es a partir de 2008 cuando se registra un crecimiento más importante. La clave de esta evolución ha sido el aumento de usuarios de las bibliotecas públicas, que pasan de 8,2 millones de inscritos en 2002 a 15,6 millones en 2012.

Alrededor del $43 \%$ de la población española está inscrita en alguna biblioteca

Los índices de préstamo se mantienen ligeramente al alza, aunque lejos del importante crecimiento conocido en años anteriores. El servicio de préstamo continúa siendo el servicio más utilizado, pero su evolución está viendo cambios en su composición y en función de la tipología bibliotecaria. Los libros impresos constituyen el tipo de documento más prestado en toda clase de bibliotecas: representan las dos terceras partes $(66,0 \%)$ de los préstamos del conjunto del sistema, un porcentaje similar al registrado en las bibliotecas públicas $(65,4 \%)$ y universitarias $(68,4 \%)$.

Durante 2012 en las bibliotecas públicas se prestó un total de 56,6 millones de documentos, un descenso de $6,1 \%$ con respecto al dato de 2010 (60,2 millones) que rompe la evolución ascendente de la última década. Ese descenso se debe especialmente a los audiovisuales (1,9 millones menos), a los documentos sonoros (casi un millón menos), a las publicaciones periódicas y a otros

Gráfico 5. Evolución del número de usuarios 2002-2012 (millones). 
documentos, mientras que el préstamo de libros se mantiene casi en el mismo valor de 2010, con un ligero ascenso. Esta desaceleración en el préstamo de libros impresos no se ha visto compensada por el préstamo de libros electrónicos, que se mantiene en niveles realmente modestos.

Dos de cada diez bibliotecas no disponen de ordenadores y terminales informáticos de uso público: hay todavía 401 bibliotecas públicas sin ellos

Una tendencia similar sigue el préstamo de documentos audiovisuales, grupo que constituye el segundo en volumen $(25,4 \%)$ tras el préstamo de libros en las bibliotecas públicas. Sin embargo, el préstamo de documentos sonoros, que en 2002 registraba valores superiores a los audiovisuales comienza desde 2006 -cuando los recortes presupuestarios no habían hecho aún su aparición - un progresivo descenso hasta situarse en 2012 en valores que representan poco más de la mitad de los recogidos en 2004, pasando de 136 documentos sonoros por cada mil habitantes a 59. Esta evolución se explica por los cambios en los hábitos de consumo de música, en los que los soportes físicos han sido sustituidos por el acceso y descargas a través de internet.

En el caso de libros y audiovisuales los descensos en el préstamo, que se producen a partir de 2012 , son posteriores al recorte en el presupuesto de adquisiciones, que ya se observó en 2010. Por lo tanto, la explicación de esta desaceleración tal vez haya que buscarla en los problemas que están teniendo las bibliotecas para la renovación de las colecciones.

\subsection{Estancamiento y capítulos pendientes en aspec- tos tecnológicos}

El nivel de equipamiento informático de las bibliotecas españolas en 2012 mantiene prácticamente los mismos índices que en 2010 y, en algunos casos, que en 2008. Estos últimos años no parecen traer una puesta al día para las bibliotecas que carecían de tales equipamientos.

Dos de cada diez bibliotecas no disponen de ordenadores y terminales informáticos de uso público y hay todavía 401 bibliotecas públicas sin ellos. La inmensa mayoría de las bibliotecas $(95,6 \%)$ dispone de ordenadores o terminales informáticos para gestión interna. La media de ordenadores conectados a internet para uso público por cada biblioteca, que en 2012 es de 7,8, apenas ha mejorado.

Nueve de cada diez bibliotecas $(89,8 \%)$ tienen acceso a internet en sus salas, un servicio casi universal en las bibliotecas públicas $(94,9 \%)$ y en las universitarias (97,9\%). Son proporciones que se registraban ya en 2010 , incluso muy parecidas a las de 2006.
En 2012 el INE registra por primera vez el número de lectores de libros electrónicos de que disponen las bibliotecas españolas: un total de 4.908 dispositivos, de los que cerca de las tres cuartas partes $(71,6 \%)$ pertenecen a bibliotecas públicas, y una quinta parte $(19,3 \%)$ a las universitarias. Hay indicios de que se trata de una herramienta de lectura concentrada en unas pocas bibliotecas: sólo 1 de cada 10 bibliotecas públicas dispone de servicios de consulta en sala de libros electrónicos.

En el capítulo de automatización, es enorme la cifra de bibliotecas sin catálogo de consulta pública (opac) en 2012, casi la mitad (46,5\%) no lo tienen. En el caso de las bibliotecas públicas el porcentaje es muy similar, $43,8 \%$.

La presencia en internet es otra de las tareas pendientes. Los datos de 2010 publicados por el INE, los últimos disponibles, señalaban que un tercio de las bibliotecas tienen web, proporción casi idéntica a la de las bibliotecas públicas. Los datos del MECD correspondientes a 2011 no indican avances en este apartado en el caso de las bibliotecas públicas.

Las bibliotecas públicas no han podido responder adecuadamente a la crisis

\section{Conclusiones sobre el estado de las bibliotecas en 2012}

Coincidiendo con los años en que se produce la crisis económica se observa una mayor demanda de los servicios bibliotecarios por parte de los ciudadanos, como queda patente a través de los índices de visitas e inscritos. Sin embargo, las bibliotecas públicas no han podido responder adecuadamente a la crisis, a las demandas que ha generado entre los ciudadanos, al menos en lo que se refiere a los servicios de préstamo, que son precisamente los más utilizados (Hábitos..., 2013). Y no han podido hacerlo por los recortes que han constreñido seriamente la renovación de sus colecciones.

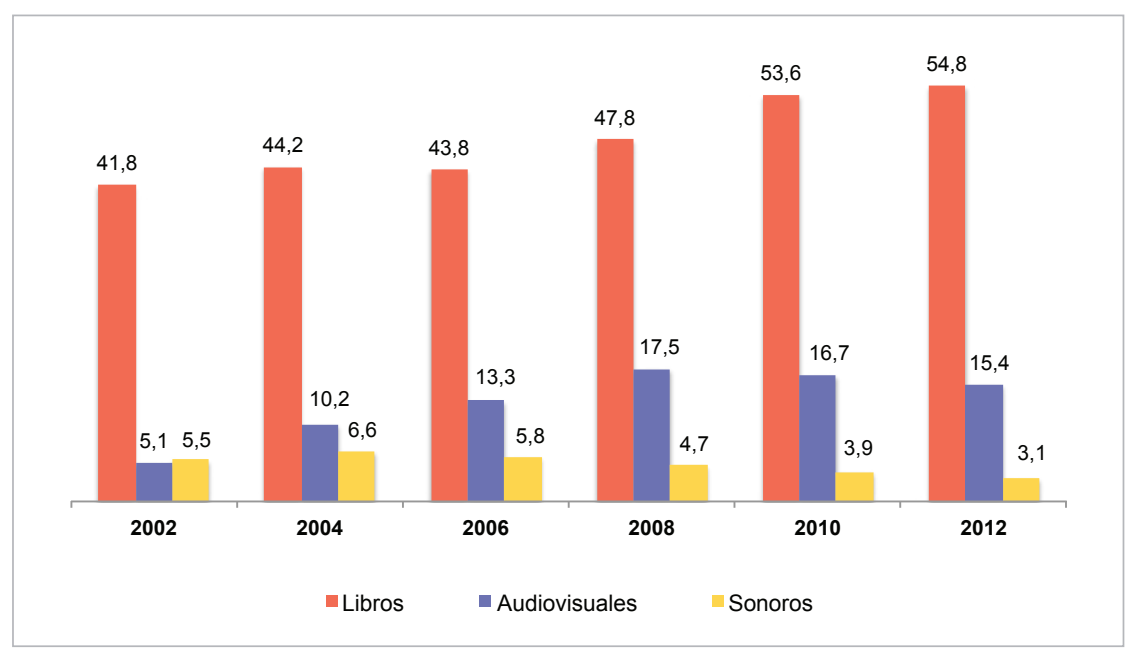

Gráfico 6. Préstamo de libros, documentos sonoros y audiovisuales, 2002-2012. 
El esfuerzo realizado durante la última década en las bibliotecas españolas se está perdiendo en algunos aspectos, en los que se vuelve a niveles de principios de siglo. Los resultados obtenidos tras ese esfuerzo muestran en 2012 los primeros signos de deterioro de la calidad de servicios como el préstamo.

Es enorme la cifra de bibliotecas aún sin opac en 2012: sólo lo tiene un 53,5\%

La crisis económica que atraviesa España está dejando huella de forma evidente en el gasto, con especial influencia en el capítulo de adquisiciones, y por lo tanto también en las colecciones, que envejecen. También en el personal, con la pérdida de puestos de trabajo, en la desaparición de puntos de servicio y en la reducción de los horarios de apertura. La tendencia descendente comenzó a notarse de forma clara en el gasto de adquisiciones en 2010 y con el tiempo se han añadido el resto de los apartados mencionados.

La distribución del gasto tiende a un cierto desequilibrio, ya que el gasto en personal alcanza niveles por encima del $70 \%$ y revierte así de manera negativa en los presupuestos para adquisiciones, actividades y otros servicios. Este desequilibrio se había detectado en las bibliotecas públicas a lo largo de la década de los noventa (Hernández-Sánchez, 2008) y había sido superado en los primeros años del siglo XXI.

En el ámbito de lo digital las bibliotecas siguen teniendo asignaturas pendientes importantes sin resolver, como los catálogos automatizados y la presencia web. Por otra parte, desde 2008 los niveles de equipamiento informático se han estancado, lo que hace pensar que existe un número de bibliotecas que no han superado la etapa pre-tecnológica y que, teniendo en cuenta la situación, no parece que la vayan a superar en los próximos años. Lo digital trae también cambios en los hábitos de consumo a los que las bibliotecas se deberán adaptar en los próximos años.

\section{Notas}

1. La serie del INE está disponible en el apartado Sociedad de su sitio web.

http://www.ine.es

2. La tasa de equivalencia se calcula dividiendo el número total de empleados entre el dato de personal en equivalencia a tiempo completo.

\section{Bibliografía}

ALA (2013). "State of America's Libraries Report 2013". American Libraries, special issue.

http://www.ala.org/news/state-americas-libraries-report-2013

Anglada, Lluís (2012). “¿Podemos hablar de crisis desde las bibliotecas?". Anuario ThinkEPI, v. 6, pp. 68-72.

Anstice, Ian (2013). "Top UK library trends of 2013". Public library news, 14 diciembre.

http://www.publiclibrariesnews.com/2013/12/top-ukpublic-library-trends-of-2013.html

Arroyo-Vázquez, Natalia; Hernández-Sánchez, Hilario; ViIloldo, Francisco J. (2012). "Bibliotecas y crisis económica (I). Las bibliotecas españolas en 2010". Biblioblog, 30 enero. http://biblioblog.org/2012/01/30/bibliotecas-y-crisis-i-lasbibliotecas-espanolas-en-2010

Carrión-Gútiez, Alejandro (2013). "Informe de situación de las bibliotecas públicas españolas en 2012". El profesional de la información, mayo-junio, v. 22, n. 3, pp. 250-258. http://dx.doi.org/10.3145/epi.2013.may.09

Federación de Gremios de Editores de España (2013). Hábitos de lectura y compra de libros en España 2012. Madrid: Federación de Gremios de Editores de España.

http://www.editoresmadrid.org/media/43692/hábitos\%20 lectura\%20año\%202012.pdf

Gómez-Hernández, José-Antonio (2010). "La acción de las bibliotecas públicas en tiempos de crisis”. Anuario ThinkEPI, v. 4, pp. 79-86.

Gómez-Yáñez, José-Antonio (coord.) (2014). El valor económico y social de los servicios de información: bibliotecas. Madrid: Fesabid.

https://www.dropbox.com/s/qff9bve9afjwmt8/EstudioFesabid_El-valor_alta.pdf

Gutiérrez-Santana, Félix; Real-Duro, Ana; BustamanteRodríguez, Antonio-Tomás; Guerrero-Salguero, Cristóbal (2010). "Estudio sobre el impacto de la crisis económica en las bibliotecas andaluzas". Boletín de la Asociación Andaluza de Bibliotecarios, julio-diciembre, n. 100, pp. 117-136.

http://www.aab.es/app/download/4555821/PDFDefinitivo-Boletín-100.pdf

Hernández-Sánchez, Hilario (dir.) (2008). Las bibliotecas públicas en España. Dinámicas 2001-2005. Madrid: Fundación Germán Sánchez Ruipérez. ISBN: 9788489384736

http://travesia.mcu.es/portalnb/jspui/bitstream/10421/1543/1/ Las_Bibliotecas_Publicas_dinamicas.pdf

Hernández-Sánchez, Hilario; Gómez-Hernández, JoséAntonio; Merlo-Vega, José-Antonio (2014). "Los profesionales de la información en España, tres años después". Anuario ThinkEPI, v. 9 [en prensa].

Merlo-Vega, José-Antonio (2013). “Bibliotecas públicas, recesión económica e inclusión social”. En: 31 Feria internacional del libro, Liber. Madrid, 3 octubre.

http://es.slideshare.net/biblioblog01/bibliotecas-pblicasrecesin-econmica-e-inclusin-social

Villoldo, Francisco J.; Hernández-Sánchez, Hilario; ArroyoVázquez, Natalia (2012). "Bibliotecas y crisis económica (II). Las bibliotecas públicas en 2010: el desarrollo de la última década, en peligro". Biblioblog, 13 febrero.

http://biblioblog.org/2012/02/13/bibliotecas-y-crisiseconomica-ii-las-bibliotecas-publicas-en-2010-eldesarrollo-de-la-ultima-decada-en-peligro 